\title{
Infección ocular invasora por Scedosporium apioespermum en un paciente inmunocomprometido
}

\author{
Invasive ocular fungal infection by Scedosporium apiospermum in an \\ immunocompromised patient
}

Renato Palma-Fernández', Alba Montecinos-Astorga ${ }^{2,6}$, Alberto Fica ${ }^{1,7}$, Patricio Godoy-Martínez ${ }^{3}$, Iván Aguilera ${ }^{4}$ y Cristóbal Pinar-Pacheco ${ }^{5}$

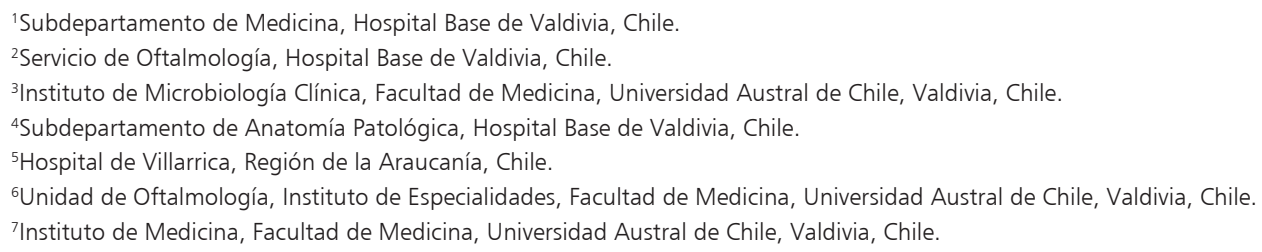

Fuente financiamiento: ninguna

Conflicto de interés: ninguno

Recibido: 6 de marzo de 2021 / Aceptado: 7 de julio de 2021

\section{Resumen}

Presentamos el caso clínico de una infección fúngica invasora con una conjuntivitis necrosante, escleritis y panuveitis unilateral por Scedosporium apiospermum en una mujer de 78 años con artritis reumatoidea con neutropenia secundaria a fármacos. El diagnóstico etiológico fue confirmado por cultivo micológico de secreción ocular con apoyo de MALDI-TOF-TOF e histopatología. El tratamiento incluyó aseos quirúrgicos asociado a terapia tópica y sistémica con voriconazol y corticoesteroides, con una evolución favorable a los dos meses de tratamiento. Una recaída obligó a un segundo curso terapéutico por 12 meses adicionales con mejoría y erradicación del agente. La conjuntivitis fúngica por $S$. apiospermum es un evento infrecuente asociado a pacientes inmunocomprometidos. Su tratamiento involucra desbridamientos quirúrgicos y terapia antifúngica prolongada.

Palabras claves: Scedosporium apiospermum; conjuntivitis; panuveitis; neutropenia; voriconazol.

\section{Abstract}

We report a case of invasive fungal infection with necrotizing conjunctivitis, scleritis and unilateral panuveitis caused by Scedosporium apiospermum in a 78-year-old woman that developed neutropenia by drugs indicated for rheumatoid arthritis. The etiological diagnosis was confirmed by mycological culture of an ocular secretion with the support of MALDI-TOF-TOF analysis and histopathological findings. The treatment involved surgical debridements together with topical solution and systemic therapy with voriconazole and steroids with a favorable evolution after 2 months of treatment. A relapse required a second therapeutic course for an additional 12 months with improvement and eradication of the agent. Fungal conjunctivitis due to S. apiospermum is a rare event associated with immunosuppressed patients. Its treatment involves surgical debridements and prolonged antifungal therapy.

Keywords: Scedosporium apiospermum; conjunctivitis; panuveitis; neutropenia; voriconazole. 


\section{Introducción}

$\mathrm{E}$ 1 aumento de pacientes inmunocomprometidos por diferentes causas ha posibilitado el desarrollo de infecciones oportunistas por una diversidad de agentes. Entre ellos, las infecciones fúngicas representan una importante causa de morbi-mortalidad debido a su complejidad diagnóstica, terapéutica y pronóstico. Scedosporium apiospermum es un hongo filamentoso de origen ambiental con resistencia intrínseca a diferentes compuestos antifúngicos y que representa una causa emergente de enfermedad fúngica en el mundo. Se asocia a infecciones invasoras o localizadas con compromiso endocárdico, pulmonar, articular, de piel y tejidos blandos, o en otras localizaciones ${ }^{1}$ Así también, se han reportado casos de conjuntivitis, coriorretinitis, endoftalmitis, queratitis o escleritis, las que pueden evolucionar a una pérdida de visión ${ }^{2-7}$. En este contexto, presentamos un caso de infección fúngica invasora (IFI) ocular por $S$. apiospermium en una paciente con inmunosupresión secundaria a fármacos. El caso estuvo caracterizado por grandes dificultades terapéuticas.

\section{Caso clínico}

Mujer de 78 años con antecedentes de artritis reumatoidea (AR) con fibrosis pulmonar de patrón usual, hipertensión arterial, diabetes mellitus tipo 2 sin requerimiento de insulina y cardiopatía coronaria con infarto al miocardio. Su terapia habitual incluía losartán, ácido acetilsalicílico y deflazacort (40 mg día de prednisona equivalente). Seis semanas antes del ingreso y con parámetros hematológicos normales, se cambió la terapia a prednisona $10 \mathrm{mg} /$ día y azatioprina $100 \mathrm{mg} /$ día por la AR activa $(1,5 \mathrm{mg} / \mathrm{kg})$. Se internó por un aumento progresivo de su disnea basal, fatigabilidad y palpitaciones. Se constató en Urgencia una hipotensión arterial de $60 / 40 \mathrm{mmHg}$ con taquiarritmia por fibrilación auricular (150/min), sin fiebre. La arritmia logró ser convertida eléctricamente recuperando su hipotensión arterial. Al examen físico segmentario se constató un aumento de volumen palpable en el tercio externo del ojo derecho (OD), hiposfagma, hematoma subconjuntival superior y queratitis ipsilateral. En el hemograma se pesquisó una pancitopenia con anemia $(\mathrm{Hb} 11,1 \mathrm{~g} / \mathrm{dl})$, trombocitopenia $(95.000$ céls/ $\left.\mathrm{mm}^{3}\right)$, leucopenia $\left(900\right.$ céls $\left./ \mathrm{mm}^{3}\right)$ y neutropenia absoluta $\left(0\right.$ céls $\left./ \mathrm{mm}^{3}\right)$. Se inició terapia antibacteriana empírica con ceftazidima, amikacina y estimulador de colonias de granulocitos junto a tobramicina tópica y lágrimas artificiales. Progresó con un marcado eritema y edema periorbitario derecho con ojo rojo, secreción conjuntival (Figura 1A) y disminución de la agudeza visual (AV). Posteriormente, miodesopsias en OD. A la exploración se observó una maceración conjuntival, cámara anterior con fenómeno de Tyndall +2 , y celularidad vítrea +1 . La tomografía axial computarizada reveló una colección periocular derecha de aspecto piógeno (Figura 1B). Se agregó tratamiento tópico con dexametasona y moxifloxacino. La neutropenia se recuperó a los seis días, luego de la suspensión de aziatioprina al ingreso. Los cultivos de sangre y orina resultaron negativos y el estudio de la médula ósea efectuado para descartar una neoplasia solo indicó cambios displásicos compatibles con una reacción adversa a fármacos. Durante la evolución se agregó disfagia, y la aparición de úlceras bucales y candidiasis oral por lo que se adicionó fluconazol y también aciclovir oral luego de confirmar una esofagitis herpética.

El cultivo de la secreción conjuntival realizado en agar papa dextrosa evidenció a los 7 días de incubación a $27^{\circ} \mathrm{C}$ el desarrollo de una colonia de aspecto algodonoso de color blanca con tonalidades de gris (Figura 3A). En

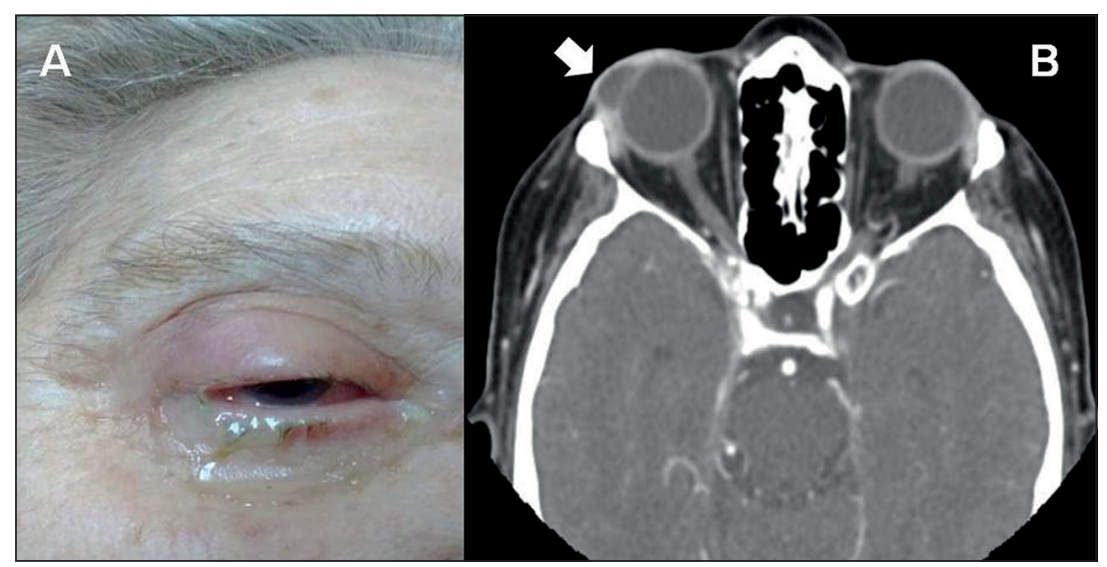

Figura 1. A: Aumento de volumen y eritema de párpado superior derecho, con presencia de secreción mucopurulenta densa en la comisura palpebral. B: Corte transversal de tomografía axial computarizada de órbita con contraste. Colección periocular derecha de aspecto piógeno (flecha blanca), destacando realce periférico con el medio de contraste.

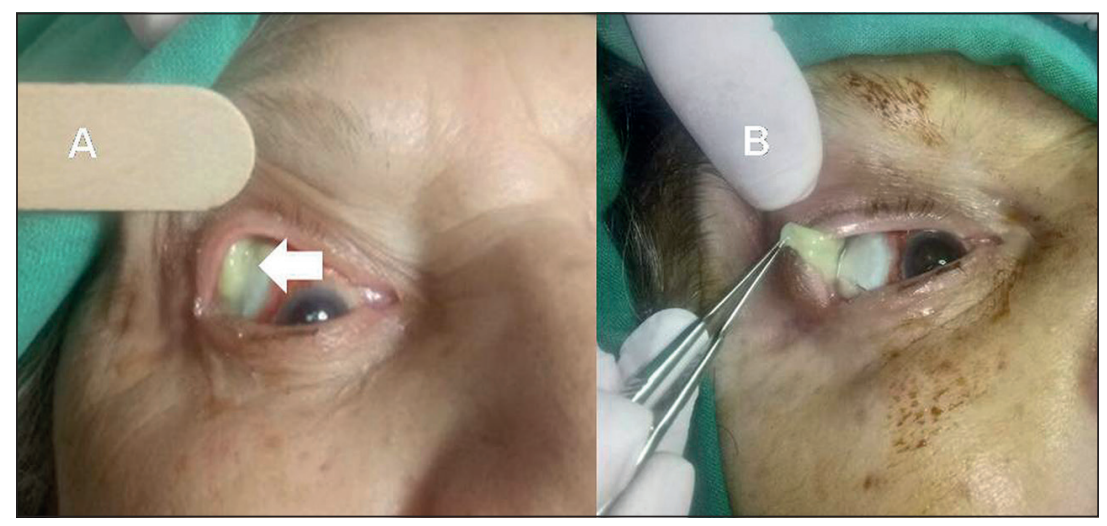

Figura 2. A: Aumento de volumen conjuntival superior en OD de aspecto desvitalizado. B: Desbridamiento de tejido conjuntival con extensión a fondo de saco. 

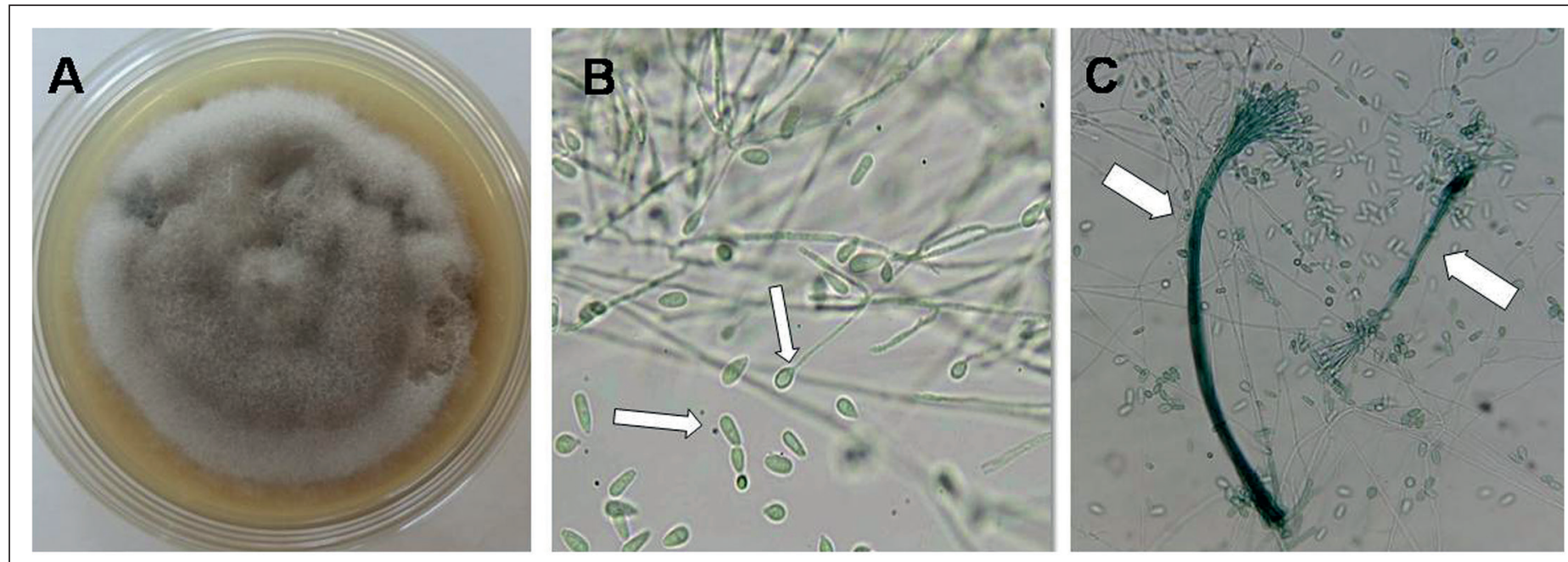

Figura 3. Cultivo de la secreción conjuntival realizado en agar papa dextrosa (BD, EE.UU) a los 7 días de incubación a $27^{\circ} \mathrm{C}$. Colonia de aspecto algodonoso de color blanca con tonalidades de gris (3A). En la micromorfología con azul de lactofenol se observaron hifas tabicadas hialinas con conidióforos no ramificados con aneloconidios de base trunca con una tonalidad más oscura compatibles con Scedosporium spp. (3B, flechas). Sinanamorfo denominado sinema que consiste en conidióforos ramificados, que terminan en anélides donde se acumulan conidios en una masa mucoide (sugiriendo S. apiospermum, 3C).

la micromorfología realizada con azul de lactofenol se observaron hifas tabicadas hialinas con conidióforos no ramificados con aneloconidios de base trunca con una tonalidad más oscura. Esta morfología fue compatible con Scedosporium spp. (Figura 3B). Además, se observó el sinanamorfo denominado sinema, que corresponden a conidióforos ramificados terminados en anélides, donde se acumulan conidios en una masa mucoide, sugiriendo la especie S. apiospermum (Figura 3C). La confirmación de este agente se realizó por espectrometría de masas MALDI-TOF-TOF MS mediante equipo AutoflexSpeed, BrukerDaltonics.

La determinación de la concentración inhibitoria mínima (CIM) fue realizada según el documento M38A2 del Clinical and Laboratory Standards Institute ${ }^{8}$ utilizando diferentes compuestos (Sigma-Aldrich, Chile). Itraconazol, voriconazol, posaconazol y caspofungina presentaron actividad sobre el hongo aislado (CIM 0,5 $\mu \mathrm{g} / \mathrm{ml}$ en todos los casos).

Se realizó un tratamiento médico-quirúrgico con aseo y desbridamiento del tejido necrótico conjuntival (Figura $2 \mathrm{~A}$ y $\mathrm{B}$ ) asociado a terapia antifúngica tópica ocular con voriconazol (solución preparada en agua bidestilada al $1 \%$ con dosis de una gota cada hora) y oral (dosis de carga habitual y luego mantención con $200 \mathrm{mg}$, dos veces al día).

Durante el primer mes evolucionó en forma tórpida con baja visión ( $\mathrm{AV}<0,05$ : sólo contar dedos), panuveitis (cámara anterior con Fenómeno de Tyndall +2 ; celularidad en vítreo anterior y visibilidad del fondo de ojo +2 ) con desprendimiento de la coroides de $360^{\circ}$ y escleritis que evolucionó a una escleromalacia, descartándose la posibilidad de terapia intravítrea por el estado escleroconjuntival. No obstante, se logró la estabilización del cuadro tras dos aseos quirúrgicos y aumento de la dosis de prednisona a $20 \mathrm{mg} / \mathrm{d}$, observando signos de reepitelización progresiva de la conjuntiva, cámara anterior sin Tyndall, disminución de la turbidez vítrea (celularidad en vítreo anterior $+0,5$ ) y reabsorción del desprendimiento coroideo con recuperación de la AV OD a 0,4.

La paciente fue dada de alta a las seis semanas con cobertura tópica con aminoglucósidos, corticoesteroides y voriconazol, recibiendo también terapia oral de mantención con este último compuesto. Los niveles plasmáticos predosis de voriconazol fueron adecuados $(5,2 \mu \mathrm{g} / \mathrm{ml}$; rango aceptable 1 a $5,5 \mu \mathrm{g} / \mathrm{ml})$. Por una buena evolución, se suspendió el tratamiento a las ocho semanas de su inicio.

Luego de un mes de suspensión de la terapia antifúngica sistémica, se detectó una recaída con signos locales de reinfección y áreas de aspecto necrosado en el lecho escleral. Se internó para reiniciar el tratamiento con voriconazol tópico y oral en las mismas dosis y un nuevo aseo quirúrgico. El cultivo micológico resultó negativo, sin embargo, el estudio histológico demostró hifas sugiriendo la persistencia del agente (Figura 4). Durante esta segunda hospitalización, evolucionó con una hiponatremia moderada atribuida en primera instancia a voriconazol, requiriendo cambio de terapia antifúngica a anidulafungina i.v. (100 mg/d), completando un mes con este compuesto. Posteriormente reinició voriconazol oral atribuyéndose la hiponatremia al uso de tiazidas. 


\section{Caso Clínico}

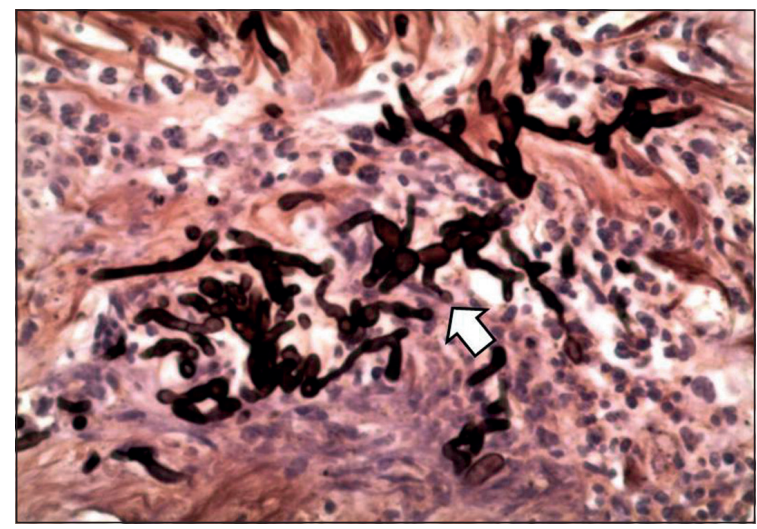

Figura 4. Estudio histopatológico microscópico del tejido necrótico en el lecho escleral por sospecha de recidiva de conjuntivitis necrosante. Tinción de Grocott (400X) muestra hifas tabicadas presentes focalmente entre el tejido fibrinoleucocitario (flecha).

La evolución fue favorable, dándose de alta a las cinco semanas. Se decidió agregar terbinafina oral $(250 \mathrm{mg} /$ día) como terapia antifúngica complementaria, pero se suspendió a las dos semanas por un exantema facial macular eritematoso. Tras observar una lesión nodular, subepitelial, grisácea sugerente de un foco micótico en el lecho escleral, se realizó una exploración quirúrgica con toma de muestras para cultivo, sin desarrollo de microorganismos. El estudio histopatológico sólo reveló depósitos secuelares de hemosiderina. La paciente se mantuvo con terapia tópica y oral con voriconazol por 12 meses adicionales, con una buena respuesta clínica (Figura 5). Su condición al alta fue favorable, logrando visiones en OD de 0,6; sin recaída tras más de dos años de seguimiento. De acuerdo a la nomenclatura estandarizada para uveítis, la paciente presentó un cuadro de inicio agudo, de duración limitada ( $<3$ meses inicialmente) pero de carácter crónico por la recaída antes de los tres meses. Sin embargo, logró finalmente una remisión completa (> 3 meses inactiva luego de suspender el tratamiento) ${ }^{9}$.

\section{Discusión}

Scedosporium apiospermum vive en ambientes con contaminación orgánica, incluyendo lugares con presencia de excrementos de aves o ganado, pantanos y aguas residuales ${ }^{1}$. Su presencia en estos lugares explica su asociación con infecciones cutáneas y su capacidad de diseminarse por conidios, permitiendo el compromiso ocular o de la vía respiratoria. La IFI del caso presentado parece estar bien respaldada por el aislamiento de un agente patógeno en los cultivos, la presencia de hifas filamentosas compatibles en el estudio histopatológico y

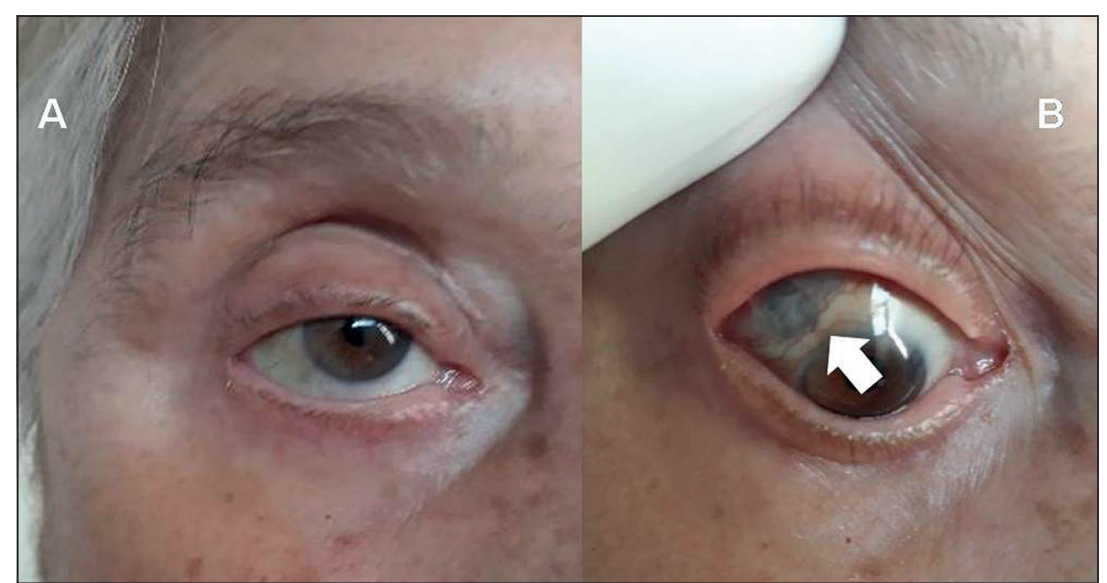

Figura 5. A-B. Seguimiento clínico a los tres meses de reinicio de terapia antifúngica sistémica sin evidencias de actividad fúngica. La flecha indica la zona comprometida con adelgazamiento de la esclera (escleromalacia) y la visualización de la coroides (zona gris).

una neutropenia intensa. Es de interés que, en este caso el diagnóstico se logró por la combinación de métodos de cultivos convencionales junto a un análisis proteómico logrado por espectrometría de masas (MALDI-TOFTOF), el que ha mejorado el reconocimiento de hongos filamentosos en el laboratorio ${ }^{10,11}$.

La mielosupresión por azatioprina ocurre aproximadamente en $5-25 \%$ de los pacientes que reciben este fármaco, se concentra en el primer año de uso y sólo rara vez $(0,9 \%)$ es potencialmente grave (neutropenia $<500$ céls $\left./ \mathrm{mm}^{3}\right)$. Se expresa como una leucopenia asociada o no a trombocitopenia o pancitopenia ${ }^{12}$. Se han descrito varios factores de riesgo para la aparición de leucopenia en los usuarios de azatioprina tales como el uso de altas dosis diarias $(>2 \mathrm{mg} / \mathrm{kg}$ ), altos niveles intracelulares de metabolitos intermediarios, ciertos polimorfismos de la enzima tiopurina S metiltransferasa (TMPT), una actividad reducida de ella o de la enzima inosina trifosfato pirofosfatasa (ITPA) y una baja actividad o ciertas variantes de la enzima Nudix hidrolasa $15^{13}$. En Chile, aproximadamente $9 \%$ de la población tiene bajos niveles enzimáticos de TMPT aunque su actividad no fue medida en nuestra paciente ${ }^{14}$. La neutropenia y linfopenia asociada a este fármaco determinaron el desarrollo de una IFI, candidiasis oral y una esofagitis herpética.

Las infecciones asociadas a S. apiospermum son pleomórficas y abarcan un amplio rango de expresiones clínicas según la inmunidad del paciente, presencia de alteraciones en la mucosa respiratoria, trauma cutáneo o procedimientos quirúrgicos. Las infecciones incluyen en general, cinco formas clínicas: micetomas subcutáneos por inoculación, colonización en dilataciones o cavidades respiratorias con mucosas alteradas previamente existentes (bolas fúngicas), compromiso sino-pulmonar 
o pulmonar invasor (sinusitis o neumonía necrosante), infecciones extrapulmonares (ósea, muscular, ocular y del SNC) e infecciones diseminadas ${ }^{1}$. Al respecto, las infecciones oculares engloban casos de queratitis, conjuntivitis, uveítis, coriorretinitis, endoftalmitis e infecciones de la órbita ocular ${ }^{1-7}$.

Las IFI por S. apiospermum son consideradas infecciones emergentes en pacientes inmunocomprometidos, especialmente en aquellos con neoplasias hematológicas o sometidos a trasplante de órganos sólidos ${ }^{15}$. Las alternativas terapéuticas para esta especie incluyen voriconazol (CIM90 0,5 $\mu \mathrm{g} / \mathrm{ml}$; rango 0,06-2 $\mu \mathrm{g} / \mathrm{ml}$ ), caspofungina (CIM90 0,5 $\mu \mathrm{g} / \mathrm{ml}$ ), anidulafungina (CIM90 $1 \mu \mathrm{g} / \mathrm{ml}$ ) o posaconazol (CIM90 $1 \mu \mathrm{g} / \mathrm{ml})^{1,16,17}$. A pesar de que terbinafina tiene una baja actividad in vitro sobre S. apiospermum (CIM $\geq 16 \mu \mathrm{g} / \mathrm{mL})$, ha sido usado en combinación con otros compuestos antifúngicos debido a un efecto sinérgico favorable ${ }^{1,18}$.

Las infecciones por S. apiospermum son difíciles de tratar y a menudo fatales en pacientes inmunocomprometidos $^{1}$. La baja frecuencia de estas infecciones no permite contar con directrices proveniente de estudios controlados y su manejo descansa en las experiencias publicadas y datos de laboratorio. La información disponible indica la importancia de revertir o controlar la inmunosupresión (reducción o suspensión del fármaco involucrado y terapia con factor estimulador de granulocitos-macrófagos), el desbridamiento quirúrgico, terapia con voriconazol (considerada como la terapia de elección), otras alternativas plausibles o la combinación sinérgica de compuestos antifúngicos ${ }^{1,11,18,19}$. En el grupo de los triazoles, voriconazol y posaconazol presentan una elevada actividad sobre este agente (rango CIM 0,01-2 $\mu \mathrm{g} / \mathrm{ml}$ y $0,03-2 \mu \mathrm{g}$ / $\mathrm{ml}$; respectivamente), teniendo las equinocandinas una actividad menor $(0,25$ a $4 \mu \mathrm{g} / \mathrm{ml}$ para caspofungina; 1 $>16 \mu \mathrm{g} / \mathrm{ml}$ para anidulafungina). En contraste, la actividad de itraconazol, anfotericina B y terbinafina es reducida y sus valores $\mathrm{CIM}_{90}$ son muy elevados (cercana o mayor a $16 \mu \mathrm{g} / \mathrm{ml}$ ) lo que impide su consideración terapéutica ${ }^{1}$. Los resultados subóptimos logrados con monoterapia antifúngica ha llevado a usar terapias combinadas sinérgicas de voriconazol con anfotericina B liposomal o terbinafina con resultados variables ${ }^{1,11}$.

En pacientes neutropénicos, el control de la neutropenia es fundamental para lograr la sobrevida del paciente ${ }^{1} \mathrm{y}$ los escasos reportes en Chile dan cuenta de la importancia del aseo quirúrgico en el control exitoso de la infección, incluso en presencia de inmunosupresión ${ }^{20,21}$. Estudios observacionales respaldan la terapia combinada de voriconazol con terbinafina, ya que las infecciones invasoras por $S$. apiospermum han presentado un mejor pronóstico usando este enfoque (terbinafina 250-500 mg/d) ${ }^{18}$. Lamentablemente, nuestra paciente presentó un exantema secundario a terbinafina, lo que impidió aplicar esta estrategia. Las reacciones alérgicas a este compuesto se han presentado en aproximadamente $2 \%$ de los casos e incluyen prurito, exantema, urticaria, eritema multiforme, lupus cutáneo, psoriasis u otras presentaciones.

En nuestra paciente, afectada progresivamente con conjuntivitis, escleritis y finalmente con una panuveitis complicada con desprendimiento de la coroides, se aplicó un enfoque médico-quirúrgico con desbridamientos quirúrgicos y terapia prolongada con voriconazol con niveles plasmáticos adecuados. La concentración con la administración oral de voriconazol alcanza un valor aproximado de $1-1,5 \mu \mathrm{g} / \mathrm{ml}$ en el humor acuoso y de $0,8-1,1 \mu \mathrm{g} / \mathrm{ml}$ en el humor vítreo y posiblemente sean equivalentes a las del plasma en casos de inflamación ocular $(2-3 \mu \mathrm{g} / \mathrm{ml})$ lo que permite alcanzar un efecto inhibitorio local22,23. La terapia tópica con voriconazol complementa el tratamiento, logrando concentraciones $>1 \mu \mathrm{g} / \mathrm{ml}$ en la cámara anterior si las gotas son aplicadas en forma frecuente (cada hora) ${ }^{24}$. En nuestro caso se utilizó transitoriamente anidulafungina por sospecha de hiponatremia asociada a voriconazol e incluso un período de terapia combinada con terbinafina. El largo tiempo de terapia (12 meses en la segunda fase) obedece a la recurrencia clínica junto a la evidencia histológica de la persistencia del agente a pesar de dos aseos quirúrgicos y dos meses de tratamiento inicial. La recaída en infecciones oculares por este microorganismo ha sido comunicada previamente junto a la necesidad de aplicar terapias prolongadas ${ }^{6}$. Un caso semejante de infección necrosante evolucionó rápidamente a un desprendimiento coroideo masivo y, finalmente, de manera precoz, a ptisis bulbi, requiriendo manejo con enucleación ${ }^{2}$.

Este caso nos enseña la importancia de la toma de cultivos en lesiones oculares en pacientes inmunocomprometidos, incluyendo el estudio de causas micológicas. La presencia de neutropenia también permite el desarrollo de IFI oculares y su manejo depende de un enfoque integrado dedicado a revertir o limitar la inmunosupresión, desbridar el tejido comprometido, incluso en repetidas ocasiones y aplicar terapia antifúngica local y sistémica por períodos prolongados.

Agradecimientos: Los autores desean agradecer a los profesionales de la Universidad de la Frontera en Temuco, Chile por su ayuda para efectuar los análisis de MALDI-TOFTOF MS con el equipo AutoflexSpeed (Bruker Daltonics), proyecto Fondequip EQM 160054, 2016. 


\section{Referencias bibliográficas}

1.- Cortez K J, Roilides E, Quiroz-Telles F, Meletiadis J, Antachopoulos C, Knudsen T, et al. Infections caused by Scedosporium spp. Clin Microbiol Rev 2008; 21: 157-97. doi: 10.1128/ CMR.00039-07.

2.- Fernández-Flores A, López-Medrano R, Fuster-Foz C. Histopathological clues in the diagnosis of fungal infection by Scedosporium in a case of endophthalmitis starting as conjunctivitis. J Cutan Pathol 2016; 43: 461-7. doi: 10.1111/cup. 12689.

3.- Kiratli H, Uzun O, Kiraz N, Eldem B. Scedosporium apiospermum chorioretinitis. Acta Ophthalmol Scand 2001; 79: 540-2. doi: 10.1034/j.1600-0420.2001.790524.x.

4.- Jain A, Egbert P, McCulley TJ, Blumenkranz M S, Moshfeghi D M. Endogenous Scedosporium apiospermum endophthalmitis. Arch Ophthalmol 2007; 125: 1286-9. doi: 10.1001/ archopht.125.9.1286.

5.- Nulens E, Eggink C, Rijs A J, Wesseling P, Verweij P E. Keratitis caused by Scedosporium apiospermum successfully treated with a cornea transplant and voriconazole. J Clin Microbiol 2003; 41: 2261-4. doi: 10.1128/jcm.41.5.22612264.2003.

6.- Todokoro D, Hoshino J, Yo A, Makimura K, Hirato J, Akiyama H. Scedosporium apiospermum infectious scleritis following posterior subtenon triamcinolone acetonide injection: a case report and literature review. BMC Ophthalmol 2018; 18: 40. doi: 10.1186/ s12886-018-0707-4.

7.- Thomas P A. Current perspectives on ophthalmic mycoses. Clin Microbiol Rev 2003; 16: 730-97. doi: 10.1128/cmr.16.4.730797.2003.

8.- Clinical and Laboratory Standards Institute. Reference Method for Broth Dilution Antifungal Susceptibility Testing of Filamentous Fungi; Approved Standard-Second Edition. CLSI document M38-A2. Wayne: Clinical and Laboratory Standards Institute; 2008.

9.- Jabs D A, Nussenblatt R B, Rosenbaum J T. Standardization of Uveitis Nomenclature (SUN) Working Group. Standardization of uveitis nomenclature for reporting clinical data. Results of the First International Workshop. Am J Ophthalmol 2005; 140: 509-16. doi: 10.1016/j.ajo.2005.03.057.

10.- Cassagne C, Normand A C, L'Ollivier C, Ranque S, Piarroux R. Performance of MALDITOF MS platforms for fungal identification. Mycoses 2016; 59: 678-90. doi: 10.1111/ myc. 12506.

11.- Ramírez-García A, Pellon A, Rementeria A, Buldain I, Barreto-Bergter E, Rollin-Pinheiro R, et al. Scedosporium and Lomentospora: an updated overview of underrated opportunists. Med Mycol 2018; 56(supp1_1): S102-25. doi: $10.1093 / \mathrm{mmy} / \mathrm{myx} 113$.

12.- Connell W R, Kamm M A, Ritchie J K, Lennard-Jones J E. Bone marrow toxicity caused by azathioprine in inflammatory bowel disease: 27 years of experience. Gut 1993; 34: 1081-5. doi: 10.1136/gut.34.8.1081.

13.- van Gennep S, Konté K, Meijer B, Heymans MW, D'Haens G R, Löwenberg M, de Boer N K H. Systematic review with meta-analysis: risk factors for thiopurine-induced leukopenia in IBD. Aliment Pharmacol Ther 2019; 50: 484-506. doi: 10.1111/apt.15403.

14.- Jorquera A, Solari S, Vollrath V, Guerra I, Chianale J, Cofré C, et al. Genotipo y fenotipo de la enzima tiopurina metiltransferasa en población chilena. Rev Med Chile 2012; 140: 889-95. doi: 10.4067/S003498872012000700009.

15.- Low C Y, Rotstein C. Emerging fungal infections in immunocompromised patients. F1000 Med Rep 2011; 3: 14. doi: 10.3410/M314.

16.- Guinea J, Peláez T, Recio S, Torres-Narbona $\mathrm{M}$, Bouza E. In vitro antifungal activities of isavuconazole (BAL4815), voriconazole, and fluconazole against 1,007 isolates of zygomycete, Candida, Aspergillus, Fusarium, and Scedosporium species. Antimicrob Agents Chemother 2008; 52: 1396-400. doi: 10.1128/ AAC.01512-07.

17.- Espinel-Ingroff A. In vitro antifungal activities of anidulafungin and micafungin, licensed agents and the investigational triazole posaconazole as determined by NCCLS methods for 12,052 fungal isolates: review of the literature. Rev Iberoam Micol 2003; 20: 121-36.

18.- Henao-Martínez A F, Castillo-Mancilla J R, Barron M A, Nichol A C. Combination antifungal therapy in the treatment of Scedosporium apiospermum central Nervous system infections. Case Rep Infect Dis 2013; 2013: 589490. doi: 10.1155/2013/589490.

19.- Tortorano A M, Richardson M, Roilides E, van Diepeningen A, Caira M, Munoz P, et al. European Society of Clinical Microbiology and Infectious Diseases Fungal Infection Study Group; European Confederation of Medical Mycology. ESCMID and ECMM joint guidelines on diagnosis and management of hyalohyphomycosis: Fusarium spp., Scedosporium spp. and others. Clin Microbiol Infect 2014; 20 Suppl 3: 27-46. doi: 10.1111/1469-0691.12465.

20.- Chanqueo L, Gutiérrez C, Tapia C, Silva V, Razeto L, Misad C. Infección rinosinusal por Scedosporium apiospermum en un hospedero inmunocompetente. Rev Chilena Infectol 2009; 26: 453-6. doi: 10.4067/S071610182009000600011.

21.- Díaz M C, Juliet C, Monzón D L T, RodríguezTudela J L. Absceso herida operatoria por Scedosporium prolificans: Primer aislamiento en Chile. Revisión de la literatura. Rev Chilena Infectol 2004; 21: 65-9. doi: 10.4067/S071610182004000100010 .

22.- Logan S, Rajan M, Graham E, Johnson E, Klein J. A case of aspergillus endophthalmitis in an immuncompetent woman: intra-ocular penetration of oral voriconazole: a case report. Cases J 2010; 3: 31. doi: 10.1186/1757-1626-331.

23.- Breit S M, Hariprasad S M, Mieler W F, Shah G K, Mills M D, Grand M G. Management of endogenous fungal endophthalmitis with voriconazole and caspofungin. Am J Ophthalmol 2005; 139: 135-40. doi: 10.1016/j. ajo.2004.08.077.

24.- Al-Badriyeh D, Neoh C F, Stewart K, Kong D C. Clinical utility of voriconazole eye drops in ophthalmic fungal keratitis. Clin Ophthalmol 2010; 4: 391-405. doi: 10.2147/opth.s6374. 